Journal of Optics

November 2015, Volume 17 Issue 11 Pages 114010

http://dx.doi.org/10.1088/2040-8978/17/11/114010

http://archimer.ifremer.fr/doc/00298/40907/

(c) 2015 IOP Publishing Ltd Printed in the UK

\title{
Comparison of adhesion layers of gold on silicate glasses for SERS detection
}

\author{
Colas Florent ${ }^{1,5,{ }^{*}}$, Barchiesi Dominique ${ }^{2}$, Kessentini Sameh ${ }^{3}$, Toury Timothée ${ }^{4}$, \\ Lamy De La Chapelle Marc ${ }^{5}$
}

1 Ifremer, LDCM, F-29280 Plouzané, France

2 Université de Technologie de Troyes, INRIA - GAMMA3 - ROSAS, F-10000 Troyes, France

${ }^{3}$ University of Sfax, Faculty of Science of Sfax, Sfax, Tunisia

${ }_{5}^{4}$ Université de Technologie de Troyes, UMR 6279, ICD-LNIO, F-10000 Troyes, France

${ }^{5}$ Université Paris 13, UMR 7244, CSPBAT, F-93000 Bobigny, France ${ }^{3}$

* Corresponding author : Florent Colas, email address : florent.colas@ifremer.fr

\begin{abstract}
:
Gold is one of the most widely used metals for building up plasmonic devices. Although slightly less efficient than silver for producing sharp resonance, its chemical properties make it one of the best choices for designing sensors. Sticking gold on a silicate glass substrate requires an adhesion layer, whose effect has to be taken into account. Traditionally, metals ( $\mathrm{Cr}$ or Ti) or dielectric materials (TiO2 or Cr2O3 ) are deposited between the glass and the nanoparticle. Recently, indium tin oxide and (3mercaptopropyl)trimethoxysilane (MPTMS) were used as a new adhesion layer. The aim of this work is to compare these six adhesion layers for surface- enhanced Raman scattering sensors by numerical modeling. The near-field and the far-field optical responses of gold nanocylinders on the different adhesion layers are then calculated. It is shown that MPTMS leads to the highest field enhancement, slightly larger than other dielectric materials. We attributed this effect to the lower refractive index of MPTMS compared with the others.
\end{abstract}

Keywords: surface-enhanced raman scattering, adhesion layer, nanocylinder, discrete dipole approximation 


\section{Introduction}

The Surface Enhanced Raman Scattering (SERS) is based on near-field enhancement of the electric field which probes the molecules in vicinity of the sensor. Therefore the most common way to improve the sensitivity of SERS setup is to excite the localized surface plasmon resonance (LSPR) of nanoparticles of noble metal. One of the most widely used metals is gold due to its high conductivity, malleability and ductility. It suffers from a slightly smaller Q-factor than silver [1] but its chemical properties make it good for building up sensors. Gold is chemically inert in aqueous environment and can be easily functionalized as it creates strong chemical bonds to moieties such as thiol or disulfide [2]. However, it adheres poorly to silicate glass. An adhesion layer is then required. The most widely spread materials are metal such as chromium or titanium and metal-oxide such as $\mathrm{TiO}_{2}$ or $\mathrm{Cr}_{2} \mathrm{O}_{3}$. Indium Tin Oxide (ITO) was also reported as a gold adhesion layer [3].

The effect of the adhesion layer on the electronic properties has been extensively studied [4]. However few works have been reported on the effect of the adhesion layer of gold based plasmonic structures and devices. In 2008, the influence of metallic adhesion layer was investigated for gold thin films [5, 6] as well as nanodisks [7, 8]. Ref. [7] clearly showed a shift and a broadening of the extinction band because of the chromium. The relative attenuation of the extinction can reach $45 \%$ for nanodisks with height smaller than $30 \mathrm{~nm}$, diameter smaller than $150 \mathrm{~nm}$ and a Cr adhesion layer of thickness $4 \mathrm{~nm}$ [8]. Recently, the effect of adhesion layers of $\mathrm{Cr}, \mathrm{Ti}, \mathrm{Cr}_{2} \mathrm{O}_{3}, \mathrm{TiO}_{2}$ and ITO on the optical properties of nanostructures has been studied. Plasmon modes of bow ties nanostructures [9] as well as the fluorescence enhancement capabilities of nanoholes in a gold layer were deeply analyzed [10]. These works demonstrated that the field enhancement is larger for dielectric than for metallic adhesion layer. $\mathrm{Ti}$ and $\mathrm{Cr}$ adhesion layers were also compared for polystyrene bead trapping by bow-ties [11]. The former showed a better efficiency and trapping stiffness than the latter. 
To overcome this disrupting effect of the metallic adhesion layer, dielectric materials are of interest, due to their low absorption of electromagnetic energy. (3-mercaptopropyl)trimethoxysilane (MPTMS) is an alkoxysilane molecule with a thiol functional group termina. This compound is a good candidate as it can be grafted to glass silicate substrate through silanization reaction while establishing strong chemical bonds with gold nanoparticles [12]. Recently, the use of MPTMS during electron beam lithography (EBL) process was reported $[13,14,15]$. The extinction efficiency of nanocylinders deposited on glass with MPTMS and without any adhesion layer were very similar [15] and much sharper than with Ti. Similar nanostructures demonstrated high SERS enhancement capabilities [13]. The authors experimentally observed an enhancement of the $1200 \mathrm{~cm}^{-1}$ band of trans-1,2-Bis(4Pyridyl)Ethylene (BPE) of one order of magnitude higher than with chromium [13]. In addition the nanocylinders strongly bind to the substrate [16]. This new adhesion layer appears particularly interesting for increasing the nanosensors gain and sensitivity without loss of robustness.

However, to our knowledge, no comparison of MPTMS with other adhesion layers was performed for SERS application. First the aim of this paper is to compare the influence of the adhesion layer on the SERS gain using different adhesion materials: metals $\left(\mathrm{Cr}\right.$ and Ti), oxides $\left(\mathrm{Cr}_{2} \mathrm{O}_{3}\right.$, ITO and $\left.\mathrm{TiO}_{2}\right)$ and MPTMS. Second, in order to provide further improvements of the SERS efficiency, we aim to give a better understanding of this effect.

\section{Material and method}

The cases of the six different adhesion layers are modeled. The SERS nanosensor consists in a square lattice arrangement of 50nm-high nanocylinders, which diameters $(\Phi)$ vary from 100 to $200 \mathrm{~nm}$ with 10nm steps (Fig. 1). The radius of curvature of the upper corner of each nanocylinder is considered equal to $10 \mathrm{~nm}$. The interparticle distance is $200 \mathrm{~nm}$. The nanoparticles lay on a glass substrate (N-BK7). The thickness of the adhesion layer is 2-nm. 
(a)

(b)
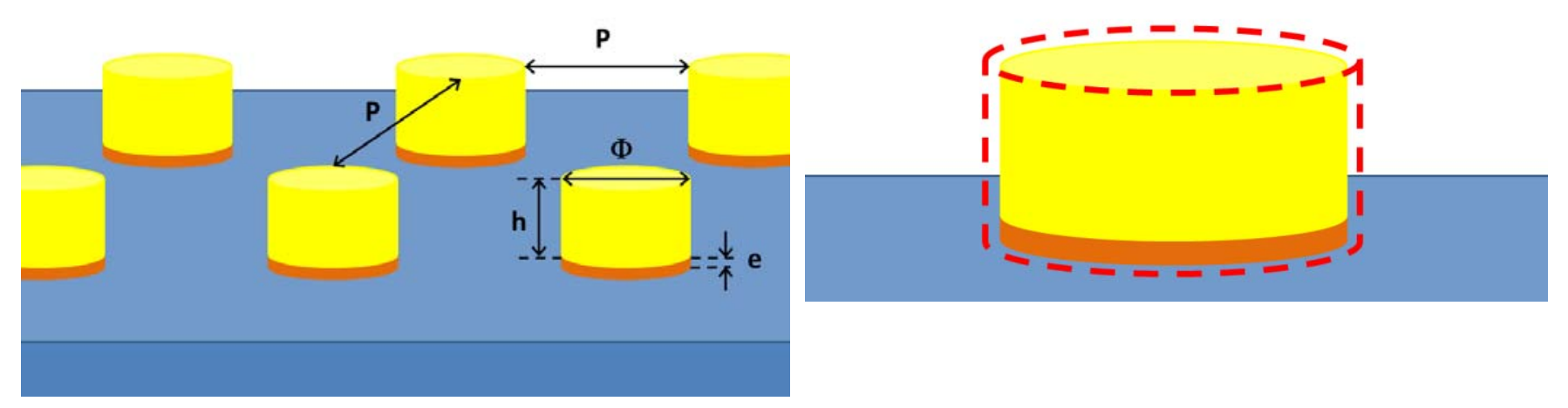

Figure 1: Schematic of the lattice of nanocylinders (a). The volume in which the intensity of the nearfield is calculated is delimited by the red dotted box and the nanoparticle outer surface (b). The field in the glass substrate is not considered.

The numerical modeling was carried out with DDSCAT 7.3 [17], this code having been proved to be efficient for plasmonic modeling [18]. Geometries are generated by home-made code written in python. The lattice size of the dipole grid is set to 2 -nm because of the memory requirements. This will limit us to adhesion layer thickness of a multiple of 2-nm. In the case of MPTMS, only a molecular layer is deposited onto the glass and the real thickness is lower. The effect of the real adhesion layer is then expected to be lower than the calculated one. A careful comparison of the theoretical results with [13] will then be carried out to assess the validity of the modeling.

The relative permittivities of the different materials are taken from different sources: Au [19], N-BK7 for Schott database, $\mathrm{Cr}$ and Ti from Sopra database, $\mathrm{Cr}_{2} \mathrm{O}_{3}$ [9], $\mathrm{TiO}_{2}$ [20], ITO [9], and MPTMS [20]. The calculations are performed with the high performance computing facilities of Pôle de Calcul Intensif pour la Mer [21].

In all cases, the extinction efficiency $\left(\mathrm{Q}_{\mathrm{ext}}\right)$ is calculated [17] as well as the mean of the electric field intensity $\left(\mathrm{E}_{\text {mean }}{ }^{2}\right)$ in a 2-nm thick shell around the upper part of the particles, excluding the glass substrate (Figure 1b). The mean of electric field intensity is calculated to predict the SERS signal. The 
SERS (g) signal is considered as proportional to the product of mean electric intensities at the laser and Raman lines:

$$
g \propto \mathrm{E}_{\text {mean }}^{2}\left(\lambda_{\text {Laser }}\right) \mathrm{E}_{\text {mean }}^{2}\left(\lambda_{\text {Raman }}\right)
$$

The computation of the electric field requires the relative permittivity of the six adhesion layers, noted $\varepsilon_{\mathrm{r}}$ and plotted on figure 2. The value of the modulus of $\operatorname{Re}\left(\varepsilon_{\mathrm{r}}\right)$ in the visible range increases for MPTMS, Cr, Ti, $\mathrm{TiO}_{2}$, ITO and then $\mathrm{Cr}_{2} \mathrm{O}_{3}$. The imaginary part of $\varepsilon_{\mathrm{r}}$ goes from 0 for MPTMS and $\mathrm{TiO}_{2}$ and then slightly increases for ITO and become large for $\mathrm{Cr}_{2} \mathrm{O}_{3}$, Ti and $\mathrm{Cr}$, in increasing order.
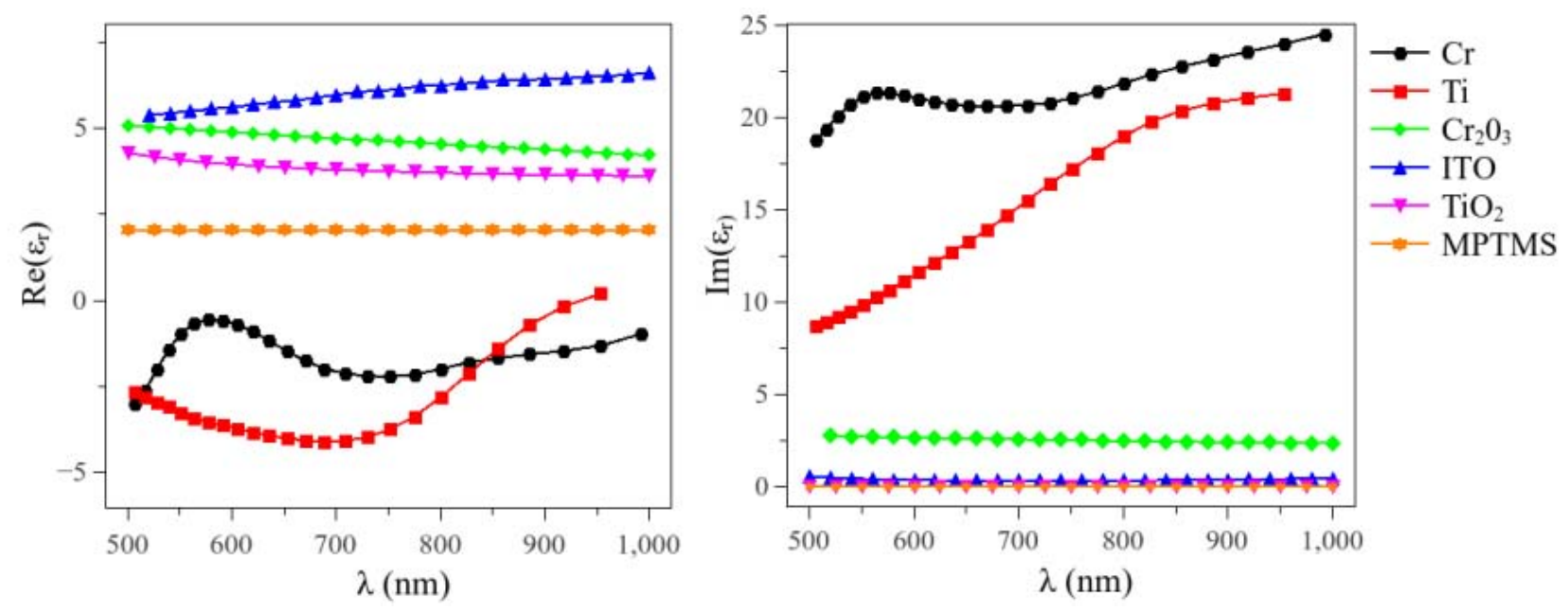

Figure 2: Real (on the left) and imaginary part (on the right) of the relative permittivity of the adhesion layers

\section{Results}

The calculated extinction spectra for $128 \mathrm{~nm}$ diameter nanocylinders are plotted on figure 3 for the six adhesion layers. The modification of the layer only induces a slight shift of the position of the extinction band with regard to each other (spectral shift lower than $30 \mathrm{~nm}$ ) as observed experimentally [13]. This is confirmed by the plots on figure 4 that shows the position of the maximum of the extinction efficiency, noted $\lambda_{\text {ext }}$, with regard to the diameter for the six adhesion layers. This shift is due to the relative permittivity of the adhesion layer on the LSPR since the higher is the modulus of $\operatorname{Re}\left(\varepsilon_{\mathrm{r}}\right)$ the more red-shifted is the extinction band, as observed experimentally [9] and predicted theoretically 
[8].

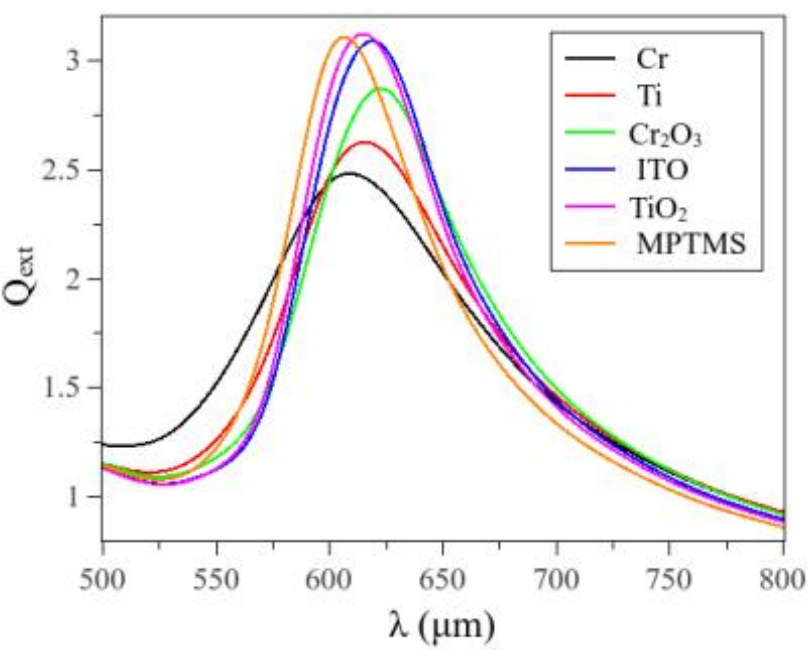

Figure 3: Q $Q_{\text {ext }}$ for a 128nm-diameter nanocylinder as a function of the wavelength.

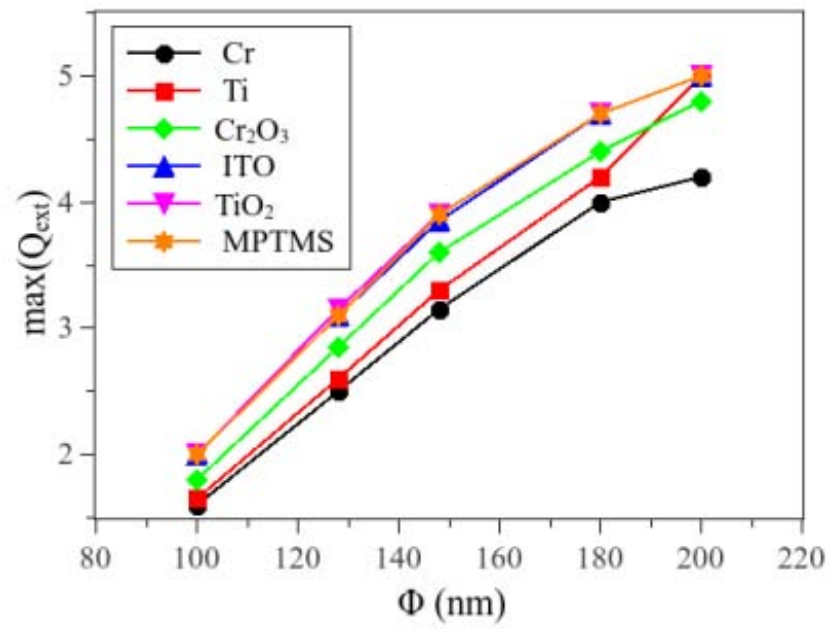

Figure 5: Maximum extinction efficiency as a function of the diameter.

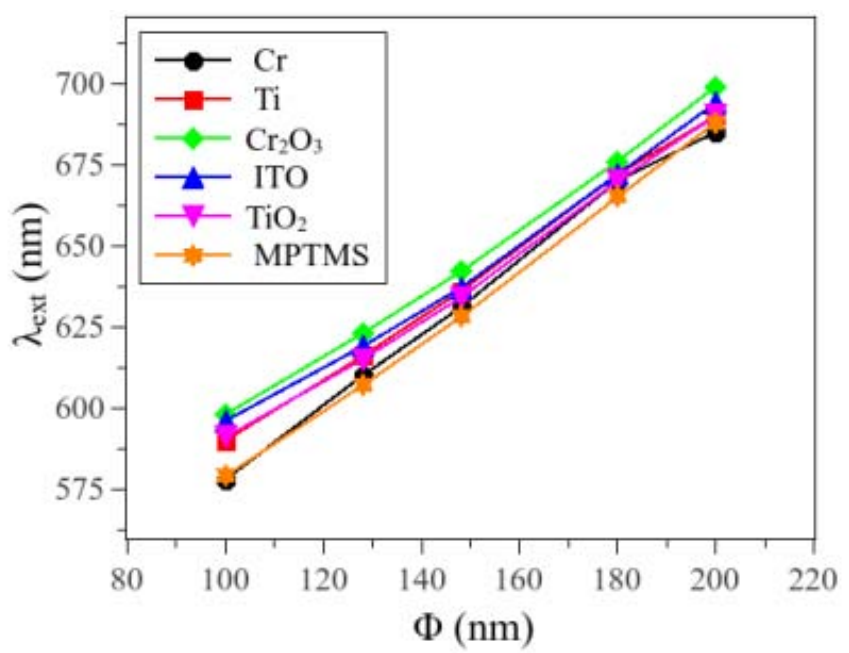

Figure 4: Position of the extinction band $\left(\lambda_{\text {ext }}\right.$ (nm)) as a function of the diameter (nm).

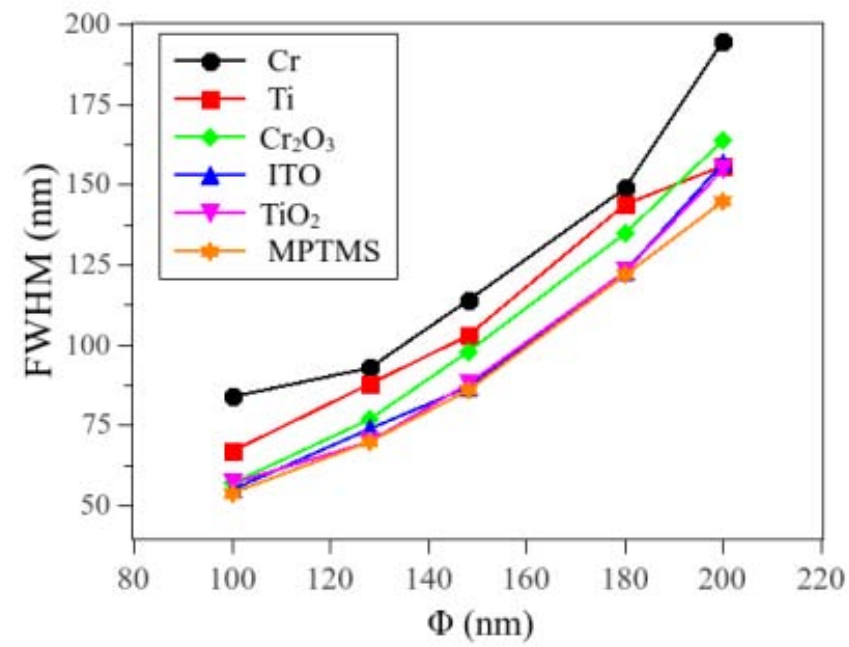

Figure 6: full width at half maximum $(F W M H)$ as a function of the diameter.

The graphs on figure 5 show the maximum of the extinction band with regards to the diameter of the cylinders in the six cases. As expected, it increases with the diameter as the scattering efficiency rises with the size of the particle. The maximum of the extinction efficiency are similar for $\mathrm{MPTMS} \mathrm{TiO}_{2}$ and ITO but regularly decreases for $\mathrm{Cr}_{2} \mathrm{O}_{3}$, Ti and then $\mathrm{Cr}$. The extinction efficiency declines as the 
imaginary part of $\varepsilon_{\mathrm{r}}$ of the adhesion layer gets larger. This decrease of the extinction efficiency cannot be neglected since it can be as large as $25 \%$ if we compare the Cr case with the MPTMS one. This absorption induces less scattering of the incoming light.

The FWHM is of particular interest for SERS applications as it relates to the sensor efficiency. The thinner is the intensity peak, the higher the maximum of the mean of intensity is (Eq. (1)).

For the six materials the FWHM increases (Figure 6) with the diameter of the nanocylinder as the radiation damping is expected to grow with the volume of the nanoparticle [22]. The FWHM rises with $\operatorname{Im}\left(\varepsilon_{\mathrm{r}}\right)$ as the losses get bigger. For instance, values of $70 \mathrm{~nm}$ and $92 \mathrm{~nm}$ are found for $128 \mathrm{~nm}$-diameter nanocylinders on MPTMS and $\mathrm{Cr}$ respectively. The numerical results are very consistent with experiments [13] equal to $65 \mathrm{~nm}$ and $98 \mathrm{~nm}$ for an equivalent diameter.

A broader extinction band leads to a lower field enhancement [13]. It is then expected that nanosensors with a dielectric adhesion layer will lead to the highest enhancement factor. This is confirmed by figure 7 that shows $\mathrm{E}_{\text {mean }}^{2}$ for a $128 \mathrm{~nm}$-diameter cylinder for the six adhesion layers. They clearly show plasmon modes, which are slightly shifted with regards to each other. The average electric fields are larger for the dielectric than for metal. For the latter, the higher $\operatorname{Im}\left(\varepsilon_{\mathrm{r}}\right)$, the lower the enhancement for all the nanocylinder diameter as confirmed by figure 8 . Figure 9 represents the maximum enhancement for the six adhesion layers with regards to the imaginary part of their permittivity. The lower the imaginary part, the higher the enhancement is. It is worth noting that the three dielectric materials are not equivalent. The enhancements are higher with MPTMS and $\mathrm{TiO}_{2}$ than ITO as the latter is slightly absorptive. However, MPTMS appears even better than $\mathrm{TiO}_{2}$. This surprising point can be explained by comparing the $\operatorname{Re}\left(\varepsilon_{\mathrm{r}}\right)$ values. MPTMS has a smaller relative permittivity than $\mathrm{TiO}_{2}$.

To illustrate the effect of the real part, the maximum enhancement for the six adhesion layers with regards to the modulus of their permittivity is plotted on figure 10. It can be clearly noted than the 
lower $\left|\varepsilon_{\mathrm{r}}\right|$ is, the higher the enhancement is. Then not only the imaginary but the real part of the relative permittivity of the adhesion layer has to be considered.

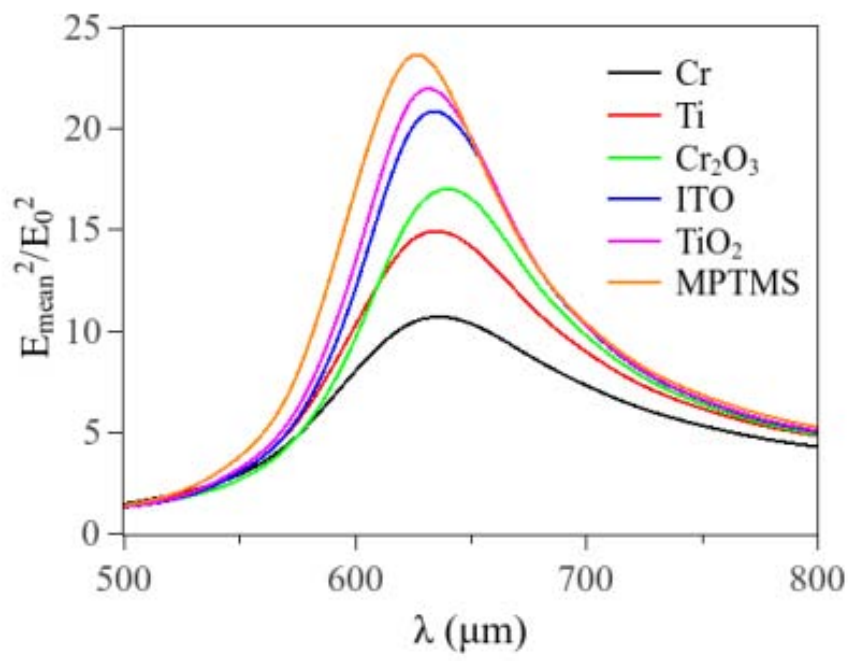

Figure 7:Near-field plasmon band of a 148nmdiameter nanocylinder

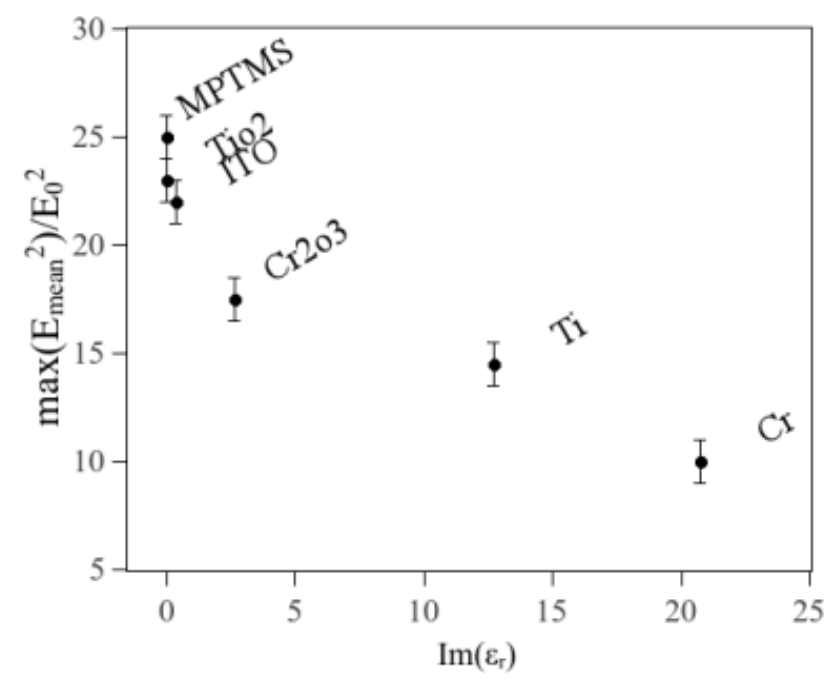

Figure 9: Maximum enhancement with regard to the imaginary part of the relative permittivity of the adhesion layer

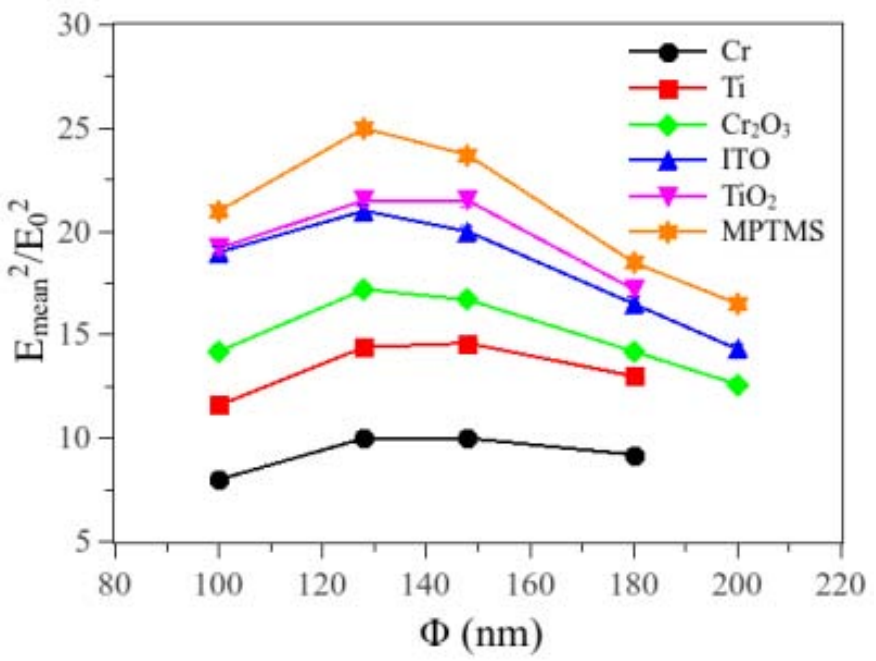

Figure 8: Field enhancement with the regards to the diameter

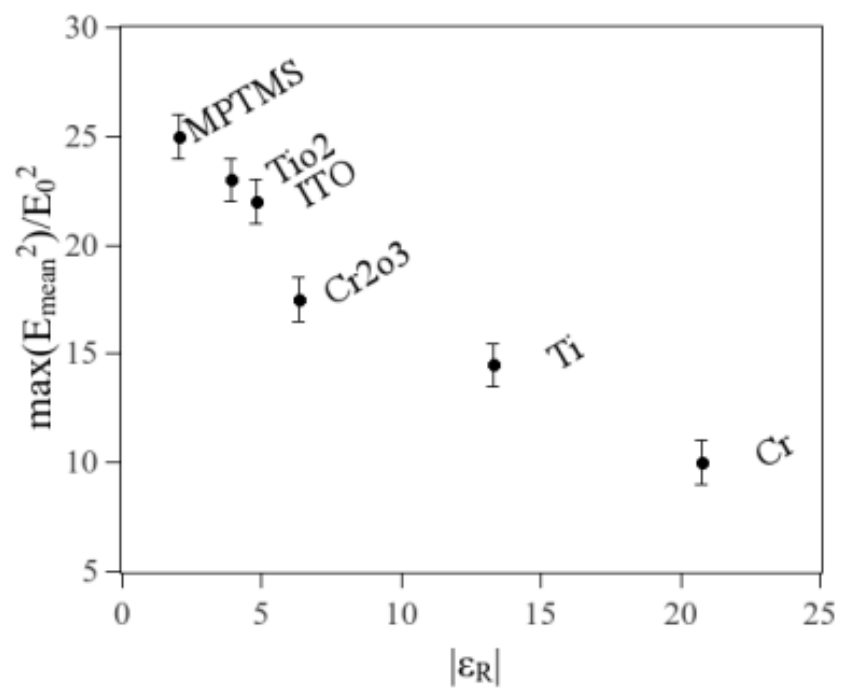

Figure 10: Maximum enhancement with regards to modulus of the relative permittivity of the adhesion layer 

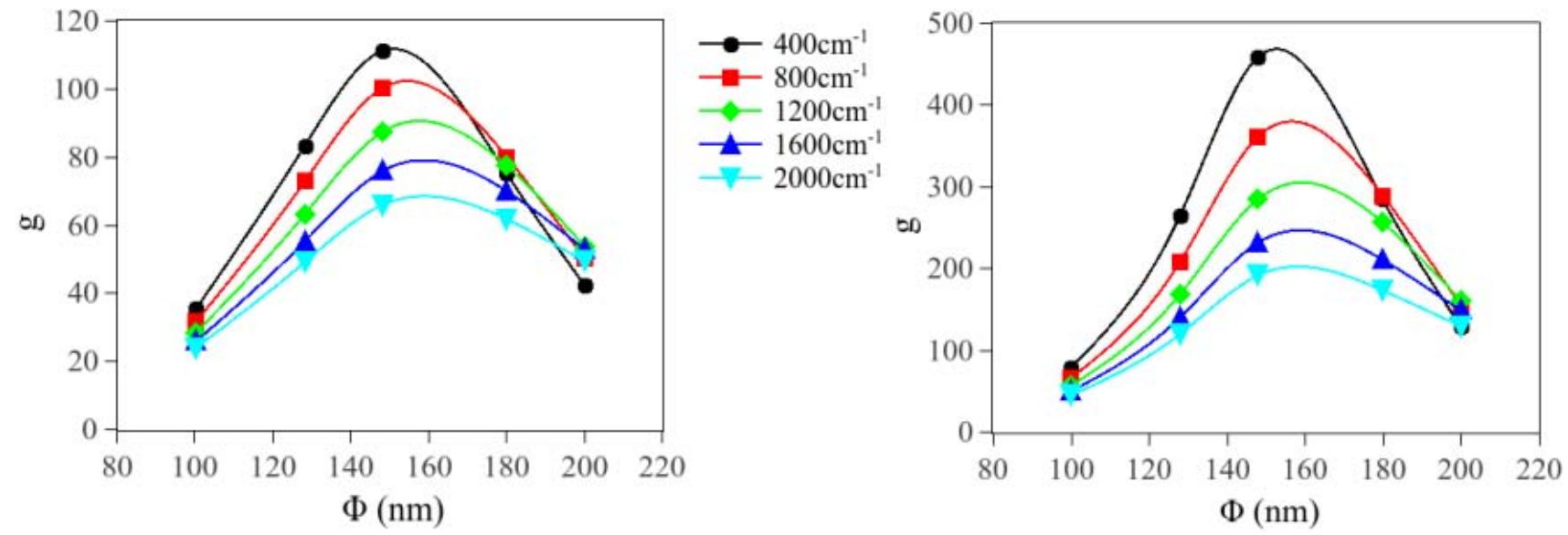

Figure 11: SERS enhancement for the nanocylinders of diameter ranging from 100 to 200nm on top of Cr (on the left) and MPTMS (on the right).

To calculate the influence of the adhesion layer on SERS signal, the mean Raman enhancements (eq. 1) was calculated for $\mathrm{Cr}$ and MPTMS as adhesion layer and Raman shifts of 400, 800, 1200, 1600 and $2000 \mathrm{~cm}^{-1}$ were calculated, with $\mathrm{HeNe}$ as laser source. The corresponding graphs are plotted on figure 11.

The best enhancement is achieved with nanocylinders of diameter of 150-160nm corresponding to an extinction band located between the laser and the Raman lines as experimentally observed [23, 24]. The larger the Raman shift, the larger the diameter maximizing the SERS enhancement. In addition, the smaller the Raman shift is, the higher the field enhancement is as both the laser line and the Raman lines get closer to the maximum field.

The enhancement value is two times lower than the experimental value measured by Shen et al [13]. This discrepancy may come from thickness difference between numerical modeling ( $2 \mathrm{~nm}$ because of the dipole grid lattice parameter) and experiment (3nm).

The same calculation with $\mathrm{TiO} 2$ showed a Raman enhancement of 10\% compare to MPTMS (data not shown) implying that this new adhesion layer demonstrated the best performance for SERS detection. 


\section{Discussion:}

To conclude on how to choose the adhesion layer, the effects of the real and the imaginary parts have to be discussed. First, chromium reduces the mean field enhancement by 2.5 -fold compare to MPTMS for nanocylinder of diameter of $128 \mathrm{~nm}$, whose resonance wavelength is about $610 \mathrm{~nm}$. Only $2 \mathrm{~nm}$ thick adhesion layer strongly affects the plasmon. Maps of the near field around a nanoparticle in the case of $\mathrm{Cr}$ and MPTMS are plotted in figure 12. It can be clearly seen that the electric field amplitude is much higher at the bottom than at the top of the nanoparticle. This was observed by Schatz and co-workers as well with silver tetrahedron [25]. At this particular location, the electric field is the superimposition of the evanescent wave associated to the Fresnel reflection at the substrate interface and the scattered wave. We assume that this hot-spot comes from the interference of these two waves.

Therefore both the electromagnetic energy and the local absorption of light are confined near the adhesion layer that plays a critical role in the whole system. This is consistent with the work reported by Jiao and co-workers on bow-ties [9] in which the absorptive layers diminished twice the field amplitude at the bottom of the nanostructure as much as at the top.

(a)
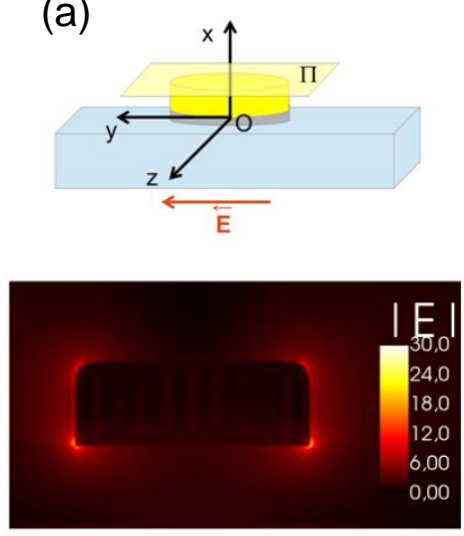

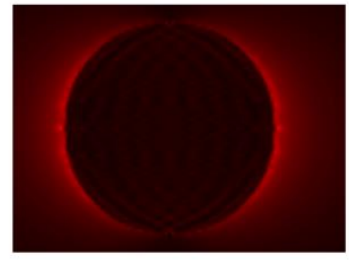

(b)
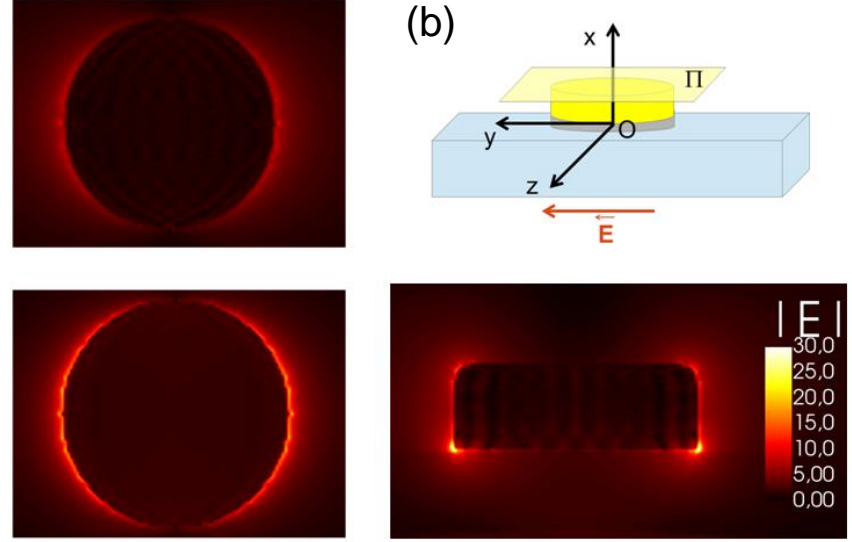
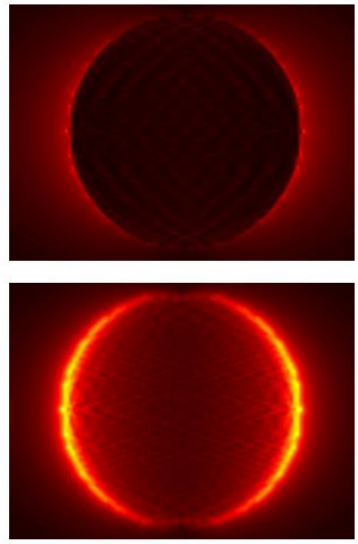

Figure 12: Maps of the modulus of the electric field in the $\mathrm{xOy}$ (bottom left), the $y \mathrm{Oz}$ (bottom right) and the Iplan (upper right) according to the coordinate system on the upper left with Cr (a) and MPTMS (b) as adhesion layer.

To understand the effect of the real part, the different dielectric materials have to be considered. To 
understand how the effect of a dielectric adhesion layer may change the enhancement of the electric field we first check if it comes from a global change of the permittivity of the nanoparticle or not.

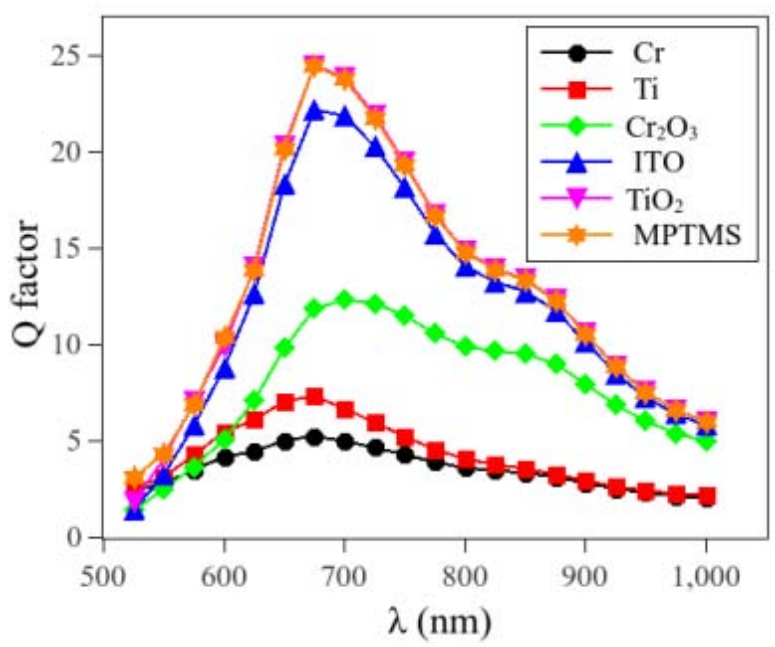

Figure 13: Q-factor of nanospheres of a mixture of gold and adhesion layer material.

To assess this assumption, the nanoparticles in the quasi-static hypothesis were modeled. For a given shape the resonance condition then depends only on the permittivity of the different materials [26] and not on the size. Then, the Q-factor is very sensitive to relative permittivity change. In addition, in the case of quasi-static nanoparticle, the Q factor is directly related to the near-field enhancement [27]. The material of the nanoparticle is considered as a mixture of gold and the adhesion material in the same volume proportion as the gold nanocylinder and the sticking layer. The permittivity was calculated using the Bruggeman model as [28] to calculate the effect of aluminium oxide on the aluminium nanoparticle properties. In this hypothesis, we consider spherical particles. The quality factor of the nanoparticle is plotted on figure 12. It was calculated as suggested in [27]:

$$
\mathrm{Q}(\omega)=\omega \frac{\frac{\partial \operatorname{Re}(\epsilon)}{\partial \omega}}{\operatorname{Im}(\epsilon)}
$$

where $\omega$ is the pulsation.

The quality factor increases from $\mathrm{Cr}, \mathrm{Ti}, \mathrm{Cr}_{2} \mathrm{O}_{3}, \mathrm{ITO}$ to $\mathrm{TiO}_{2}$ and $\mathrm{MPTMS}$ respectively (Figure 13). The quality factors of small nanoparticles are much more affected by a change of the permittivity. A five 
folds increase is calculated when using $\mathrm{TiO}_{2}$ and MPTMS instead of chromium. $\mathrm{TiO}_{2}$ and MPTMS show similar quality factor. Therefore the difference calculated by discrete dipole approximation does not come from a global permittivity change of the nanoparticle but from a local effect.

On the other hand, a paper studying the effect of the adhesion layer on delocalized SPR generated in thin films [29] demonstrates that the electric field enhancement does not change significantly when using $\mathrm{TiO}_{2}$ and MPTMS even for thickness as large as $200 \mathrm{~nm}$.

The different behavior of $\mathrm{TiO}_{2}$ and MPTMS then comes from the nanostructuration of the adhesion layer. The nanodisks formed by the adhesion layer scatter the incoming light before reaching the gold nanoparticle, which leads to a decrease in the efficiency of the plasmon coupling. We then assume that the highest enhancement is achieved when the refractive index of the adhesion layer matches that of the glass.

The modeling takes only into account the electromagnetic contribution. As we saw, the maximum enhancement is located at the bottom part of the nanocylinder. Then the interaction of the adhesion layer with the molecule is located at the hot-spot. The chemical role of this layer might be a very efficient mean of increasing the sensitivity of SERS sensors. For instance, higher hydrophobicity of MPTMS than other linker was demonstrated an efficient way of concentrating molecules for increasing the sensor sensitivity [12].

\section{Conclusions}

In this paper, the far-field and the near-field response of gold nanocylinders in a square lattice arrangement were compared for six adhesion layers of different materials. The FWHM of the extinction band depends on the imaginary part of the relative permittivity while the position depends more on the real part of the relative permittivity of the adhesion layer. It was then showed that the fieldenhancement is determined not only by the imaginary part but also by the real part. We found that the best enhancement is achieved when using dielectric material of permittivity close to that of the glass 
substrate. Absorptive materials reduce strongly the field enhancement whereas the MPTMS appeared as the best dielectric layer for SERS enhancement. The effect of the adhesion layer is particularly important as it is located at the bottom of the nanoparticle where the electric field is the highest. Moreover this location open new prospects as molecular layers were proven efficient for concentrating molecules to be detected. 


\section{References:}

[1] Le R E and Etchegoin P 2008 Principles of Surface-Enhanced Raman Spectroscopy and Related Plasmonic Effects (Oxford: Elsevier)

[2] Löfås S and Mc Whirter A 2006 Surface Plasmons Resonance Based Sensors ed J Homola and O S Wolfbeis (Heidelberg: Springer) pp 117-51.

[3] Kambayashi M, Zhang J, and Oyama M 2005 Crystal Growth \& Design 5 (1) 81-84

[4] Poate J M, Tu K M and Mayer J W (ed) 1978 Thin Films—Interdiffusion and Reactions (New York: Wiley-Interscience)

[5] Barchiesi D, Macías D, Belmar-Letellier L, van Labeke D, Lamy de la Chapelle M, Toury T, Kremer E, Moreau L and Grosges T 2008 Applied Physics B 93 177-181

[6] Sexton BA, Feltis BN and Davis TJ Sensors and actuators A 2008141 471-475

[7] Zheng YB, Juluri BK, Mao XL, Walker TR and Huang TJ 2008 Journal of Applied Physics D 103(1) 014308.

[8] Kessentini S and Barchiesi D 2013 State of the Art in Biosensors-General Aspects ed T Rinken (Rijeka: InTech)

[9] Jiao X, Goeckeritz J, Blair S and Oldham M 2008 Plasmonics 4(1) 37-50

[10] Aouani H, Wenger J, Gérard D, Rigneault H, Devaux E, Ebbesen TW, Mahdavi F, Xu T and Blair S 2009 ACS Nano 3(7) 2043-2048

[11] Roxworthy BJ and Toussaint KC 2012 Optics express 20(9) 9591

[12] Péron O, Rinnert E, Lehaitre M, Crassous P and Compère C 2009 Talanta 79(2) 199-204

[13] Shen H, Guillot N, Rouxel J, Lamy de la Chapelle M and Toury T 2012 Optics Express 20(19) $21278-21290$

[14] Lamy de la Chapelle M, Shen H, Guillot N, Frémaux B, Guelorget B, and Toury T 2013 Plasmonics8(2):411-415 
[15] Habteyes TG, Dhuey S, Wood E, Gargas D, Cabrini S, Schuck PJ, Alivisatos AP and Leone SR 2012 ACS nano 6(6) 5702-5709

[16] Guelorget B, Toury T, Shen H and Lamy de la Chapelle M 2012 Scripta Materialia 67 515-518

[17] Draine B and Flatau P 1994 Journal of the Optical Society of America A 11(4)1491-1499.

[18] Hao E, Schatz G and Hupp J 2004 Journal of Fluorescence 14(4) 331-341[19] Johnson PB and Christy RW Physcal Review B 1976 (12) 4370-4379

[20] He G, Zhang LD, Li GH, Liu M and Wang XJ Journal of Physics D: Applied Physics 41 045304045312

[21] http://www.ifremer.fr/pcim.

[22] Wokaun A, Gordon JP, and Liao PF, 1982 Physical Review letters 48(14), 957-960.

[23] Guillot N, Shen H, Frémaux B, Péron O, Rinnert E, Toury T and Lamy de la Chapelle ML 2010 Applied Physics Letter, 97(2) 023113-023113.

[24] Felidj N, Aubard J, Levi G, Krenn JR, Hohenau A, Schider G, Leitner A and Aussenegg FR 2003 Applied Physics Letter 82(18) 3095-3097.

[25] Haes AJ, Zou S, Schatz GC and Van Duyne RP 2004 J. Phys. Chem. B 108, 109-116

[26] Wokaun A 1984 Solid State Physics 38 223-294

[27] Wang F and Shen YR 2006 Physical Review Letter 97206806

[28] Knight MW, King NS, Liu L, Everitt HO, Nordlander P and Halas NJ 2014 ACS Nano 8 (1) 834840

[29] Barchiesi D, Grosges T, Colas F and Lamy de la Chapelle M 2015 J. Opt. 17114009 